\title{
Los usos tautológicos de lo psicosocial en los procesos de intervención en Colombia*
}

\section{Tautological uses of the psychosocial intervention processes in Colombia}

\author{
John Harold Vásquez Campos** \\ ORCID: 0000-0002-1646-2037 \\ Universidad Cooperativa de Colombia \\ Nelson Molina Valencia*** \\ ORCID: 0000-0002-0250-251X \\ Universidad del Valle, Colombia
}

Recibido: 14 de noviembre de 2017

Revisado: 16 de enero de 2018

Aceptado: 24 de marzo de 2018

\section{Resumen}

El presente trabajo tuvo como objetivo evaluar el El artículo se deriva de una investigación que buscaba identificar y describir los discursos acerca de lo psicosocial en los procesos de intervención en Colombia. Se analizaron 98 documentos, clasificados en: a) documentos del Estado; b) documentos de organizaciones No Gubernamentales; c) documentos teóricos de Intervención Psicosocial y d) documentos teóricos sobre lo psicosocial. Se asumió la Psicología Social como perspectiva disciplinar y la noción de discurso como elemento teórico y metodológico. Se utilizó el análisis de contenido categorial para obtener las categorías de análisis de los documentos disponibles en bases de datos científicas y en sitios web de instituciones estatales. Los resultados se centran en la categoría adjetivo. Se encontró que el uso de la palabra psicosocial como un adjetivo "intercambiable", configura expresiones ambiguas y poco claras para el entendimiento de la noción, creando un efecto redundante en su comprensión. Es necesario

Artículo de investigación. Este trabajo presenta resultados de la Investigación denominada Claroscuros de Lo Psicosocial, una controversia que no se cierra en Colombia. Universidad del Valle. Cali, Colombia. DOI: https://doi.org/10.15332/s1794-9998.2018.0002.08

Correspondencia, John Harold Vásquez Campos. Facultad de Psicología. Universidad Cooperativa de Colombia. Dirección Postal: Carrera 73 No. 2 A - 80. Correo Electrónico: john.vasquez@correounivalle.edu.co

Instituto de Psicología-Universidad del Valle. Correo electrónico: nelson.molina@correounivalle.edu.co 
que exista claridad conceptual acerca de lo psicosocial en los procesos de intervención en Colombia, declarando las bases teóricas que sustentan el uso de la noción.

Palabras clave: Psicosocial, Intervención, Intervención social, Construcción social, Tautología, Estudio documental.

\section{Abstract}

The article presents a study that aimed to identify and describe discourses about psychosocial components in intervention processes in Colombia. We analyzed 98 documents, classified as: a) State documents; b) Documents by Non-Governmental Organizations; c) Theoretical Documents on Psychosocial Intervention; and d) Theoretical documents on psychosocial components. Social Psychology was assumed as a disciplinary perspective and the notion of discourse as a theoretical and methodological element. Categorical content analysis was used to obtain the categories of analysis of the documents available in scientific databases and on websites of state institutions. The results focus on the adjective category. It was found that the use of the word psychosocial as an adjective "interchangeable", configures ambiguous and unclear expressions for the understanding of the notion, creating a redundant effect on their understanding.

Keywords: Psychosocial, Intervention, Social intervention, Social construction, Tautology, Documentary study.

\section{Introducción}

Las condiciones sociales que con el pasar del tiempo y de las épocas se configuraron en Colombia, han estado marcadas por la exclusión, la marginalidad y la vulneración social. En este escenario, las discusiones que se estaban dando en la Psicología social y la generación de políticas públicas desde el Estado, permitieron el surgimiento de una perspectiva denominada Psicosocial que, de acuerdo con algunas lecturas aplicadas y teóricas, aborda las problemáticas desde una doble dimensión, la psicológica y la social.

La aparición de lo psicosocial a consideración de Alvis (2009), se produce una vez que se ha superado el conflicto entre "lo psicológico y lo sociológico". No obstante en la Psicología Social, en el contexto de la intervención a problemáticas sociales, se identifican dos perspectivas. En la primera, lo psicosocial se entiende desde una relación dual entre lo psicológico y lo social. Autores como Ayestarán (1996), San Juan (1996), Blanco (2007) y Alvis (2009) consideran lo psicosocial en una relación de tensión entre aspectos tanto psicológicos como sociales o estructurales. Esti- man que el propósito de la intervención que se denomine psicosocial se produzca en términos del cambio social, la solidaridad y el bienestar. Así mismo, que la operacionalización (lo psicosocial traducido en acciones) está dada por un proceso de mediación entre las personas y las estructuras sociales en el que se espera que la intervención de una cree efectos en la otra.

En la segunda perspectiva se plantea la integración de las dos dimensiones. Moscovici (1986) propuso que para superar la relación dicotómica entre lo psicológico y lo social, debía entenderse lo psicosocial como una forma de abordaje para estudiar, para explicar la realidad social y propuso la existencia de una "Visión Psicosocial". Autores como Ibáñez (1987) y Molina (2012) se apartan de una consideración dual en la relación individuosociedad y atienden a una noción de lo psicosocial enfocada en el carácter procesual de las entidades sociales y los significados que se construyen en la relación con las personas a través del discurso. El discurso desde esta postura es el fundamento para entender lo psicosocial, puesto que es a partir del lenguaje como se construye y define 
la realidad. Así, la realidad no es ni psicológica, ni social, sino que se comprende en la producción discursiva que integra estas dos dimensiones.

Aunque desde la psicología social se han podido detectar estas dos perspectivas para entender lo psicosocial, en el contexto de las intervenciones que se adjudican esta noción, no es clara la relación con la disciplina ni con el cuerpo teórico que las sustenta. En la literatura sobre lo psicosocial no es fácil encontrar una conceptualización del término. Villa (2012 citado en Aya Angarita \& Laverde Gallego, 2016) indica que lo psicosocial aún carece de claridad metodológica y epistemológica a pesar de las discusiones dadas y que lo psicosocial puede ser tomado como una "moda" a la que hay que atender y un concepto que frecuentemente se incluye en los proyectos de intervención social o comunitaria.

Un ejemplo de esto es que en libros especializados en Intervención psicosocial como el de Blanco (2007), Musitú (1993) y San Juan (1996), los autores utilizan un espacio reducido para discutir conceptualmente esta noción y dedican la mayor parte del libro para desarrollar experiencias de intervención de todo tipo y con marcos conceptuales diversos.

En el contexto de las publicaciones sobre el tema, se encuentran documentos "etiquetados" como Intervención Psicosocial y esta noción es asociada de manera libre con otros conceptos otorgándole una propiedad como lo haría un adjetivo. Por ejemplo, en lineamientos técnicos del Instituto Colombiano de Bienestar Familiar (ICBF), se requiere en el perfil del Director que sea "profesional del área psicosocial". En otros apartes del documento se cita el término desarrollo psicosocial o incluso se pide que se apliquen "los principios de cuidado psicosocial” (ICBF, 2006). No obstante, no se precisa lo que significan estas palabras (área, desarrollo o cuidado) cuando son acompañadas (adjetivadas) de la palabra psicosocial.

En la misma vía y desde diferentes contextos de enunciación, los autores asocian lo psicosocial con variedad de conceptos. Gonzales y Ruiz (2010) refieren la expresión "entorno psicosocial" que no remite a un lugar claro y sus características fun- damentales. Macías, Amar y Jiménez (2005) usan reiteradamente no sólo la expresión "problemas psicosociales" sino también el de "capacidades psicosociales" sin clarificar a qué se refieren con ellas. En documento del Ministerio de Protección Social MPS (2006) se encuentran expresiones como: impacto psicosocial, trabajo psicosocial, acción psicosocial, quehacer psicosocial, perspectiva psicosocial, necesidades psicosociales, efectos psicosociales, poniendo en evidencia, un lugar común e inespecífico para aquello que es denominado psicosocial.

En suma, lo psicosocial aparece como una categoría llamativa para los interventores, los científicos sociales, gobiernos y para la comunidad en general. El uso del que suele ser objeto entrevé que es un elemento útil (tal vez indispensable en el contexto de la intervención), que le confiere al objeto con el cual se asocia una cualidad, una característica específica. Sin embargo, menos que indicar cuál es la característica principal que se confiere a lo que es denominado psicosocial, se dificulta más su entendimiento.

Con base en estos antecedentes, se vislumbró entonces la necesidad de investigar cómo se ha entendido lo psicosocial en los procesos de intervención. Para este propósito, se utilizó como marco la psicología social y el discurso como "un conjunto de prácticas lingüísticas que mantienen y promueven ciertas relaciones sociales" (íniguez Rueda \& Antaki, 1994, p.63). El discurso es entendido como una práctica social y por esto remite hacia la acción humana, teniendo como función la construcción de realidad. Su marco permitió entender lo psicosocial y las relaciones que estas construcciones lingüísticas instituyen en las intervenciones que se hacen en Colombia haciendo uso de la categoría. Por consiguiente, en esta investigación se propuso identificar y describir los discursos existentes acerca de lo psicosocial en los procesos de intervención en Colombia y así, aportar a la discusión que actualmente se desarrolla en el país. Una discusión que supera la esfera de lo académico y trasciende, como se ha venido mostrando, la aplicación de políticas públicas tan importantes como el Programa de Atención Psicosocial y Salud Integral a Víctimas PAVSIVI. 


\section{Método}

\section{Diseño}

Esta investigación se inscribió bajo un paradigma cualitativo y se basó en el Análisis de Contenido Categorial, que de acuerdo con Bardin (1986) "funciona por operaciones de descomposición del texto en unidades, seguidas de clasificación de estas unidades en categorías, según agrupaciones analógicas" (p.119). Se utilizó el análisis temático, mediante su aplicación a discursos directos (significados manifiestos) y simples. Los pasos de la técnica se sintetizaron de acuerdo con Vázquez (1994) en: 1) partir de los datos textuales (textos); 2) Descomponer los datos textuales en unidades y 3) Agrupar las unidades en categorías siguiendo el criterio de analogía, es decir teniendo en cuenta las similitudes existentes entre las unidades en función de criterios preestablecidos, según los objetivos de investigación y/o los objetivos del análisis.

\section{Técnicas de recolección y Análisis de Información}

\section{Rastreo y recuperación de documentos}

Se realizaron operaciones de búsqueda, detección y selección de documentos para conformar el cuerpo documental objeto de análisis, mediante el establecimiento de palabra clave: "Intervención psicosocial". Los bases de datos consultadas fueron PubMed, ISIWeb, Scielo y Redalyc, así como también en la plataforma académica del motor de búsqueda Google y en los sitios web de algunas instituciones estatales en Colombia. De igual manera se contó con documentos facilitados por otros investigadores y cualquier material que aportara información provechosa para los objetivos de la investigación. Los criterios de inclusión de los documentos se remitían a todos los documentos que existieran en la red y que trabajaran lo psicosocial en el contexto de intervención entre el año 2012 al 2014 en Colombia. De allí el alcance exploratorio y descriptivo de la investigación.

\section{Procedimiento de análisis}

Fase 1. Análisis Textual. Se utilizó la herramienta tecnológica AtlasTi versión 5.0 para crear una unidad hermenéutica. Mediante la opción de búsqueda automática del software se detectaron las ocasiones en las que la palabra psicosocial aparecía en los documentos. Se seleccionaron las ubicaciones que permitían realizar un análisis del contexto en el cuál se encontraba la palabra, eliminando las que se encontraban en las cornisas de los documentos y la bibliografía.

Fase 2. Análisis Contextual. Partiendo de los datos textuales, se analizó la palabra psicosocial en su contexto (ya fuera que apareciera al final de una frase, en un subtítulo o al inicio de un párrafo), lo cual originó una primera clasificación de citas que daban cuenta del significado manifiesto en el texto dentro de unidades de análisis, por ejemplo "lo psicosocial es una estrategia de intervención”.

Fase 3. Categorización de I nivel. Es un procedimiento que consistió en agrupar las unidades de análisis en categorías siguiendo el criterio de analogía, es decir se tienen en cuenta las similitudes que existan entre las unidades.

Fase 4. Categorización de II nivel. Consistió en un proceso de reducción de los datos que aumenta el nivel de abstracción y permite la creación de categorías más amplias y complejas. Implicó el análisis de los elementos al interior de las categorías, creando relaciones entre las mismas y construyendo esquemas (networks) para sintetizar la información. De estos procedimientos se desprenden los resultados finales que dan paso a los resultados y a la discusión.

\section{Resultados}

Inicialmente se incluye el cuerpo documental conformado según la búsqueda y selección de los documentos; luego el cruce de las categorías de análisis por tipos de documentos y posteriormente los resultados de la categoría Adjetivo. 


\section{Cuerpo documental conformado}

El material documental seleccionado se agrupó de acuerdo con el tipo de documento en: 1) documentos que remitieran a una experiencia de Intervención denominada psicosocial concreta. 2) documentos que hicieran una reflexión teóri- ca acerca de la intervención psicosocial. 3) documentos institucionales de entidades públicas o privadas que refirieran una experiencia de intervención psicosocial. 4) documentos de diversa índole que se refirieran a procesos de intervención psicosocial. En la tabla 1 se establece el orden en el cual se organizaron los documentos.

Tabla 1.

Distribución y cantidad de documentos del Corpus Documental.

\begin{tabular}{lcc}
\hline \multicolumn{1}{c}{ Tipo de Documento } & Rango de Organización & Cantidad \\
\hline Documentos de Intervención Estatal (D.E). & Desde el 1 hasta 48 & 48 \\
\hline Documentos de Intervención Ongs. (D.O). & Desde el 49 hasta 58 & 10 \\
\hline Artículos Teóricos Intervención Psicosocial D.T.I). & Desde el 59 hasta 82 & 24 \\
\hline Artículos Teóricos lo Psicosocial (D.T.P). & Desde el 83 hasta 98 & \multirow{2}{*}{16} \\
\hline Total documentos analizados & & \\
\hline
\end{tabular}

Fuente: Elaboración propia.

\section{Categorías de Análisis}

En el proceso de análisis llevado a cabo, se determinó la frecuencia con la que apareció la palabra psicosocial en el cuerpo documental, arrojando como resultado 2162 ocasiones. Luego de realizar la codificación se seleccionaron 809 fragmentos (citas) en torno a la palabra psicosocial que a su vez fueron agrupados en 12 categorías, de las cuales se escogieron 4, las que mejor respondían a los objetivos de la investigación y que se presentan en la tabla 2.

Tabla 2.

Categorías de Análisis de la investigación.

\begin{tabular}{llc}
\hline \multicolumn{1}{c}{ Categoría } & Definición & No. Citas \\
\hline Adjetivo & $\begin{array}{l}\text { Esta categoría incluye aquellas expresiones que están acompañadas } \\
\text { de la palabra psicosocial y en las que ella actúa como un adjetivo. }\end{array}$ & 402 \\
\hline Conceptualización & $\begin{array}{l}\text { Incluye las aproximaciones teóricas que se hacen de lo psicosocial } \\
\text { en los documentos. }\end{array}$ & 152 \\
\hline Meta & $\begin{array}{l}\text { Incluye los propósitos que guían lo psicosocial en los procesos de } \\
\text { intervención. }\end{array}$ & 71 \\
\hline Operacionalización & $\begin{array}{l}\text { Se incluye la información que indique como lo psicosocial en las } \\
\text { intervenciones es operacionalizado. }\end{array}$ & 108 \\
\hline
\end{tabular}

Fuente: elaboración propia. 


\section{Cruce de categorías de análisis por tipos de documentos}

La frecuencia de las citas correspondientes a las categorías de análisis en relación con cada grupo de documentos se incluye en la tabla 3.

Tabla 3.

Frecuencia de citas por tipos de documentos versus categorías de análisis.

\begin{tabular}{lccccc}
\hline \multicolumn{1}{c}{ Categorías } & Estado (48) & $\begin{array}{c}\text { Ongs y otras } \\
(\mathbf{1 0})\end{array}$ & $\begin{array}{c}\text { Teóricos } \\
\text { Intervención (24) }\end{array}$ & $\begin{array}{c}\text { Teóricos } \\
\text { Psicosocial (16) }\end{array}$ & Total (98) \\
\hline Adjetivo & 214 & 67 & 75 & 46 & 402 \\
\hline Conceptualización & 24 & 45 & 69 & 14 & 152 \\
\hline Meta & 17 & 7 & 38 & 9 & 71 \\
\hline Operacionalización & 37 & 15 & 46 & 10 & 108 \\
\hline Total & 292 & 134 & 228 & 79 & 733 \\
\hline
\end{tabular}

Fuente: elaboración propia.

El grupo de documentos del Estado es el que más citas le aporta al análisis, especialmente para la categoría Adjetivo que supera con amplio margen a los demás grupos de documentos. Esta situación se podría dar por el gran número de documentos que son analizados en este grupo. Sin embargo, en las otras categorías es el grupo de documentos teóricos sobre la intervención psicosocial el que más citas posee. Esto indica que la cantidad de documentos en cada grupo no influyó de manera significativa en el número de citas aportados a cada categoría, puesto que aunque cada grupo de documentos se ocupa de lo psicosocial, la aparición de las categorías no se da de la misma manera.

\section{Resultados de la Categoría Adjetivo}

La categoría Adjetivo emerge del cuerpo documental al observar la frecuencia (402 veces) y la forma como lo psicosocial aparecía en los textos. Generalmente, estuvo acompañando una palabra para darle un atributo, para otorgarle una cualidad, es decir cumplía con la función gramatical de un adjetivo. Fue frecuente encontrar cómo en las subcategorías, la palabra psicosocial se usaba para intentar designarle un sentido diferenciador a la noción que estaba acompañando. La información que se incluye a continuación acerca de las subcategorías Intervención y abordaje relacionadas con la palabra área y con la noción de integralidad, pretende ilustrar la manera como lo psicosocial ha sido utilizado como adjetivo en los procesos de intervención.

En la subcategoría Intervención, lo psicosocial pretende fungir como un adjetivo para designar acciones profesionales que se diferencian de otro tipo de intervenciones que no son psicosociales. Acciones dirigidas a personas o comunidades para ayudarles a alcanzar un mejor nivel de vida, superar alguna circunstancia adversa y que afecta su desarrollo o que le sirva para alcanzar su bienestar. Así, por ejemplo, para el Ministerio de la Protección Social (2009) (Doc. 18) la Intervención Psicosocial es una:

Estrategia y procesos sociales basados en medidas de reparación diseñadas con participación de las víctimas y en referencia a las violaciones de derechos específicas que 
la afectaron, que busca lograr cambios a una nueva situación de las víctimas que restablezca o mejore la situación anterior a las violaciones de derechos vividas (p.9).

Es decir, se desarrollan acciones desde entidades del Estado para restablecer los derechos de las personas que están inmersas en una problemática y a su vez, tienen la posibilidad de participar en un proceso.

También ocurre con la subcategoría Abordaje que incorpora expresiones como Mirada, Sentido, Visión, Perspectiva y Lectura Psicosocial. Así, se evidencia en el Departamento Nacional de Planeación (2012) (Doc. 38) que indica cómo la intervención para que sea integral debe considerar "los ámbitos individuales, familiar y comunitario, con el fin de mitigar su sufrimiento emocional, contribuir a la recuperación física y mental y, a la reconstrucción del tejido social en sus comunidades" (p. 49).

En este sentido, se encontró el interés de vincular la noción psicosocial desde lo integral porque se considera necesario en los procesos de intervención. Jaramillo, 1998 (citado por Vasco, 2012) señala que:

Cada vez más se busca que los programas de atención a víctimas sean integrales, por lo cual la inclusión de un componente psicosocial es considerado no solo como deseable, sino también fundamental; esta coyuntura hace que aumente el número de programas y proyectos psicosociales realizados por el Estado, por las ONG o por la cooperación internacional (p. 8).

Dicha vinculación de los psicosocial a los procesos de intervención, de acuerdo con Rincón et al. (2006) ha sido un proceso lento en su implementación, pero que al ser nombrado trata de atender un requerimiento legal, en donde, apostarle a la integralidad en la intervención, implica la inclusión de lo psicosocial "La legislación colombiana, desde la aprobación de la Ley 100 de 1993, reglamentó la atención integral de la EMQ-C. No obstante, el desarrollo de programas de atención integral que incluyan lo psicosocial ha sido lento" (p.44). Incluso se advierte en algunas expresiones encontradas en los documentos que la inclusión de la perspectiva psicosocial es una necesidad que no se ha cubierto en los programas de intervención.

Al respecto, González Rocha y Ruiz Campos (2010) en un texto de la Corporación Universitaria Minuto de Dios hace una crítica (en el contexto de la intervención que mencionan) debido a la inexistencia de proyectos que trabaje con las personas en "el área psicosocial y proyectos de vida de las víctimas, pues se refleja que sus vidas han quedado afectadas y destruidas, además estas personas no cuentan con ningún proceso de acompañamiento en los vínculos afectivos, familiares y social" (p. 6). Se deduce que existe una preocupación por la implementación de proyectos que contemplen lo psicosocial, como si fuera una necesidad primordial o un deber ser porque es un elemento primordial para lograr una mejor intervención.

En el orden de las anteriores formas de adjetivación ilustradas, aparece también un uso tautológico de lo psicosocial que se ilustra en la siguiente cita del Departamento Nacional de Planeación (2012) (Doc 38):

El Programa de Atención Psicosocial y Salud Integral a Víctimas atenderá los daños psicosociales y en la salud de las víctimas ocasionados o relacionados con el conflicto armado, en los ámbitos individual, familiar y comunitario, con el fin de mitigar su sufrimiento emocional, contribuir a la recuperación física y mental y, a la reconstrucción del tejido social en sus comunidades (p. 49).

En donde, se observa cómo el ámbito de atención es definido por la propia intervención. Es decir, la atención psicosocial atiende los daños psicosociales que se presentan en las personas, familias, y comunidades fruto de su condición de víctimas de la violencia. De tal manera que en esa dinámica discursiva en la que se enuncia lo psicosocial como un adjetivo, se presentan relaciones entre subcategorías que conforman una estructura circular y tautológica que puede apreciarse en la figura 1. 
Figura 1. Esquema de la Categoría Adjetivo.

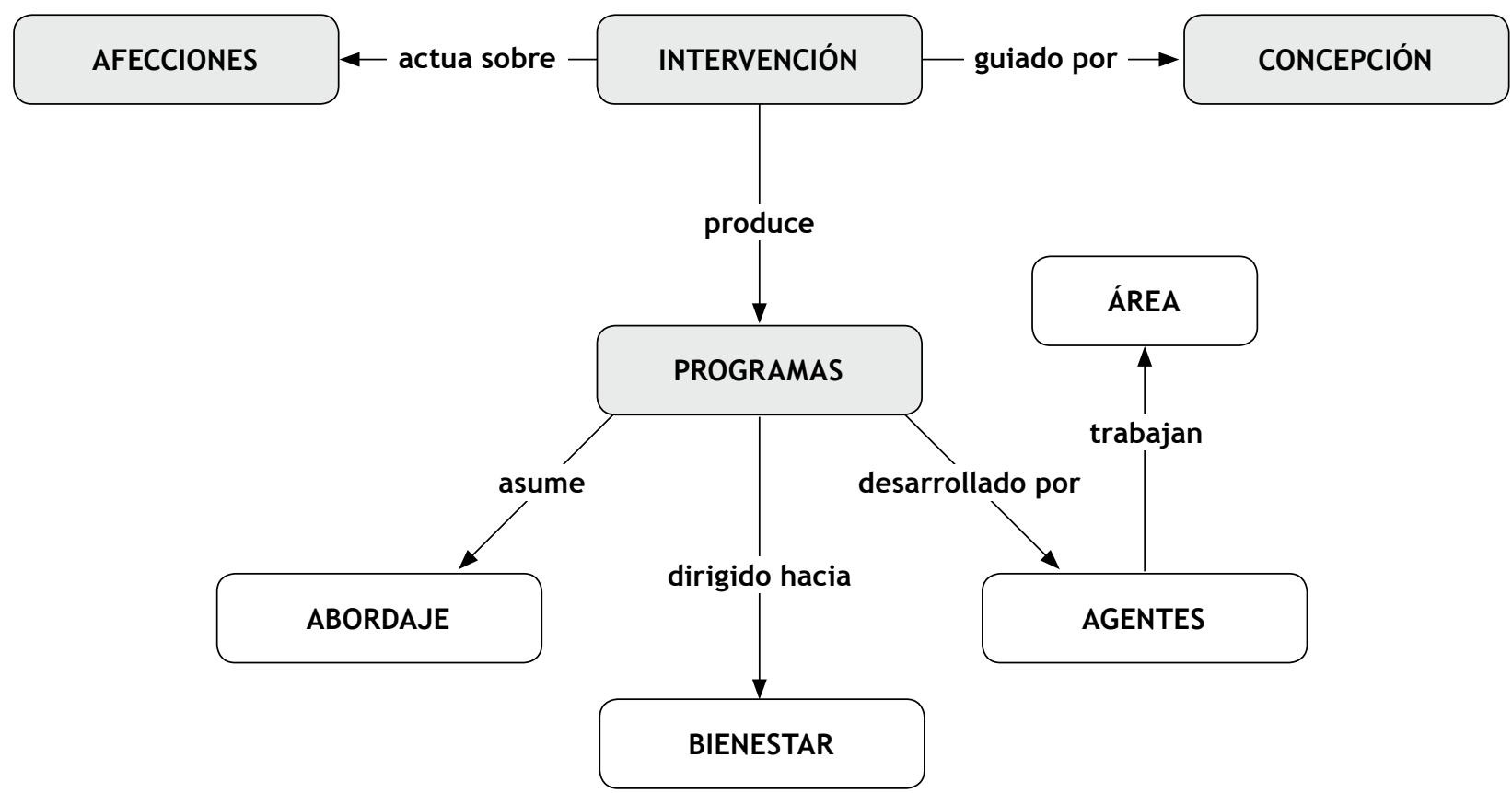

Fuente: elaboración propia.

De esta forma, el análisis del cuerpo documental en la categoría Adjetivo indica la enunciación de problemas o afectaciones psicosociales en diferentes contextos. Éstos, son afrontados desde el Estado o por otras organizaciones en la búsqueda de una mitigación o solución permanente. Para esto se implementa una intervención psicosocial que desarrolla programas psicosociales, que asumen un abordaje psicosocial y son operados por agentes o profesionales psicosociales. Éstos trabajan en el área psicosocial y buscan el bienestar psicosocial y la calidad de vida de los participantes en la intervención psicosocial.

\section{Discusión}

El discurso que construye lo psicosocial a partir de adjetivaciones constantes, hace parte de la respuesta al objetivo de identificar y describir los discursos acerca de lo psicosocial en los procesos de intervención en Colombia.

Con frecuencia se encuentra que la palabra es usada para intentar designarle un sentido diferenciador a la noción que está acompañando. Para ilustrar esta situación se incluyeron los ejemplos de subcategorías como Intervención y Abordaje.

Lo psicosocial es adjetivado en la Intervención para distinguir las acciones profesionales denominadas psicosociales. No obstante, una pregunta que surge en éste punto es ¿en qué se diferenciaría éste tipo de intervenciones con las que se hacen desde otras disciplinas y que se engloban en la denominada Intervención Social? De acuerdo con Montenegro (2001) la Intervención Social implica como idea principal que "frente a una demanda social, la acción de ciertas personas preparadas profesional y técnicamente producirá como consecuencia bienestar en las personas que son afectadas por dichas intervenciones - clientes, usuarios/as, beneficiarios/as, personas de la comunidad" (p. 69). Incluso, ¿por qué no equipararla con los fundamentos de la Investigación Acción Participativa (IAP)? Que, si se asume su enfoque investigativo, desarrolla estrategias de acción profesional para ayudar a la resolución de problemáticas en donde las personas afectadas (llámese víctimas) pueden participar. 
Una de las conclusiones que se pueden empezar a derivar del uso que se está dando a la palabra psicosocial en este caso, es que el efecto diferenciador que se busca al utilizarla, no se produce. No se ocasiona porque al tipo de intervención que se describe como psicosocial se le podría nominar desde otras posturas teóricas y disciplinas.

Esta situación también se ilustra en la subcategoría Abordaje. Pretende dar cuenta de una forma específica de entender la realidad que supone considerar aspectos personales, individuales y psicológicos, así como prestar atención a aspectos sociales y del contexto en busca de integralidad en el proceso de intervención.

Sin embargo, existen otros abordajes que también incluyen las dimensiones individual y social en su práctica. Desde un abordaje comunitario por ejemplo, se entiende que la acción profesional que busca el fortalecimiento y el bienestar de la comunidad, reconoce la importancia de entender la realidad social desde los saberes que se conjugan en la experiencia personal, grupal y comunitaria. El abordaje comunitario, en éste sentido responde a "un modelo transaccional, por cuanto hay aspectos del ambiente que afectan las acciones fortalecedoras, influyendo así en los individuos y grupos cuyas acciones a su vez pueden modificar el ambiente." (Montero, 2003, p.81).

De forma similar, desde la Intervención Social también se asume la vinculación de los procesos personales con los sociales, planteándose el reto de la integración de estos a partir del discurso y su resignificación. Según Carballeda (2002) la intervención en lo social requiere la búsqueda de una

construcción de una modalidad discursiva diferente, determinada ahora por el sujeto, por su propia palabra, por su singularidad, a la vez que recupera la importancia de los vínculos de ese sujeto con otros, buscando desde allí una resemiotización de aquello que se construyó discursivamente como hegemónico (p.111).

Así, la diferencia que se ha querido introducir al usar la palabra psicosocial asociada con Abordaje, queda desvirtuada en la medida que se pueden encontrar desde otras perspectivas los elementos que se buscaban mostrar como novedosos o de pertenencia a una perspectiva particular como la llamada psicosocial. En este caso tener en cuenta que para hacer una intervención integral se deben considerar aspectos individuales y sociales.

En relación con la noción de integralidad ligada frecuentemente a lo psicosocial en la intervención, se genera la expectativa de una ganancia adicional a la hora de intervenir con víctimas del conflicto armado si se incluye lo psicosocial, puesto que aparece como un adjetivo que le confiere integralidad a la intervención.

De esta manera, se constituye lo integral como una de las formas de comprender lo psicosocial en los procesos de intervención. Una noción que es usada con la pretensión de acercarse de mejor forma al conocimiento de la realidad, porque procura abarcar más dimensiones (individual, grupal, social, comunitaria) en las que están vinculadas las actuaciones humanas, aunque no lo consiga en sí mismo y parezca más un intercambio de palabras.

En este orden de cosas y en el escenario que se configura sobre lo psicosocial en los procesos de intervención, se puede entender por un lado que la indefinición de lo psicosocial y más aún su uso indiscriminado y acrítico ha traído como efecto no solo ambigüedad conceptual alrededor de las nociones que éste acompaña, sino que su uso se vuelva tautológico.

\section{Conclusiones}

\section{Usar lo psicosocial supone mayor efectividad en la intervención}

En lo que atañe a la categoría adjetivo, se puede concluir a partir de los datos analizados que lo psicosocial es usado con alta frecuencia en los procesos de intervención en Colombia como un elemento que quiere significar una condición de intervención diferente. Es relacionada con el término integral, que por lo general hace referencia a una intervención que al asumir la mayor cantidad de dimensiones en la acción humana 
(personal, grupal, comunitaria) tiene mayores resultados en términos de beneficios para el objeto de intervención. Es un uso que por un lado se lee como un requisito que debe ser implementado y por otro como una condición deseable e imprescindible para la intervención.

\section{La intervención denominada psicosocial se presume diferente}

La diferencia que se intenta establecer con otras intervenciones no se logra y se produce un efecto de ambigüedad e inespecificidad en los conceptos. Las nociones que se configuran no se diferencian de otras posturas teóricas (ejemplos: Intervención psicosocial y abordaje psicosocial) y se crea confusión al acompañar un concepto con la palabra psicosocial sin antes haber realizado una definición clara del mismo. La investigación muestra que la forma como es usado el concepto no le otorga un elemento diferenciador a la intervención, sino que su uso indiscriminado, promueve la naturalización de la categoría cuando es usada en el contexto de las intervenciones sociales.

\section{El uso de lo psicosocial como una Tautología}

Dada la multiplicidad de ámbitos y la frecuencia con la que es usado lo psicosocial, se contribuye a que no exista una especificidad en el término. Lo cual conlleva a una falta de claridad conceptual acerca de lo psicosocial en los procesos de intervención y en documentos académicos. Derivado del uso indiscriminado en el contexto de la intervención se produce un efecto redundante alrededor del concepto. Las definiciones que se encuentran responden a una tautología en la que un concepto es definido en términos de él mismo como se aprecia en la figura 1.

\section{Las posibilidades que brindan los marcos teóricos existentes}

Como corolario de la forma como se ha venido utilizando lo psicosocial, se favorece la realización de intervenciones sin un marco teórico evidente.
Estas consecuencias, influyen en la forma como se abordan las problemáticas sociales y los modos de acción llevados a cabo por los interventores o científicos sociales. Surge allí la posibilidad de volver a los marcos teóricos que se han construido desde las ciencias sociales. Específicamente de la psicología social, que en diálogo con otras disciplinas podría aportar a subsanar las consecuencias que se están presentando por el uso dado a lo psicosocial y que se ha ilustrado en este artículo.

\section{Referencias}

Alvis, A. (2009). Aproximación teórica a la intervención psicosocial. Poiésis, 9(17). doi: https://doi.org/10.21501/16920945.189

Aya Angarita, S., \& Laverde Gallego, D. (2016). Comprensión de perspectivas psicosociales en Colombia. Diversitas, 12(2), 201-216. Doi: https://doi.org/10.15332/s1794-9998.2016. 0002.03

Ayestarán, S. (1996). Prólogo. En C. Guillen (Ed.). Intervención Psicosocial (pp. 9-13). Barcelona: Anthropos, Editorial del Hombre.

Bardin, L. (1986). El análisis de contenido. Madrid, Akal.

Blanco, A. \& Valera, S. (2007). Los Fundamentos de la Intervención Psicosocial. En: Blanco, A. \& Rodríguez, J. Intervención Psicosocial. Madrid. Ed. Pearson.

Carballeda, A. J. (2002). La Intervención en lo social: exclusión e integración en los nuevos escenarios sociales. Buenos Aires. Editorial Paidós.

Comisión Nacional de Reparación y Reconciliación CNRR (2011). Metodologías de Intervención Psicosocial a víctimas en clave de reconciliación. Bucaramanga Sede Regional Nororiente.

Constitución Política de Colombia. 1991. Gaceta Constitucional No. 116 de 20 de julio de 1991 
González Rocha, S. \& Ruiz Campos, J. (2010). Acompañamiento psicosocial a hombres y mujeres que han sido víctimas de crímenes de Estado. (Trabajo de grado no publicado Corporación Universitaria Minuto de Dios). Recuperado de http://www.catedralibremartinbaro.org/pdfs/ACOMPANAMIENTOPSIC OSOCIALAVICTIMASDEVIOLENCIAPOLITICA. pdf

Flores-Pons, G \& Íñiguez Rueda, L. (2009) Análisis del discurso tecnocientífico que construye la muerte encefálica en un manual de coordinación de trasplantes. Discurso \& Sociedad, 3(4), 682-713.

Departamento Nacional de Planeación. Plan Nacional de Atención y Reparación Integral a Víctimas, 2012. Bogotá D.C.: Departamento Nacional de Planeación. Recuperado de https://www.dnp.gov.co/LinkClick.aspx?fileti cket=tbxvV6M0DGg\%3D\&tabid $=1080$

Fundación Universitaria Luis Amigó FUNLAM (s.f). Aproximación a la Intervención Psicosocial. Recuperado el 10 de Octubre de 2012, de: http://virtual.funlam.edu.co/repositorio/ sites/default/files/AproximacionalalntervencionPsicosocial.pdf

Garay, A., Iñiguez, L. \& Martínez, L. (2005). La perspectiva discursiva en psicología social. Subjetividad y procesos cognitivos, 7, 105-130.

Gonzales, S \& Ruiz, J (2010). Acompañamiento psicosocial a hombres y mujeres que han sido víctimas de crímenes de estado. Recuperado el 10 de Octubre de 2012 de http://hdl.handle.net/10656/858

Ibáñez, T. (1987). La "mirada" psicosocial "emergente" y su aplicación al estudio de una categoría social como por ejemplo la juventud [versión electrónica]. Revista de la Facultat de Lletres de la Universitat de Girona, 7, 73-84.

Instituto Colombiano de Bienestar Familiar. República de Colombia (2006, Junio). Lineamientos técnicos. Modalidades hogares infantiles
- lactantes y preescolares. Recuperado el 29 de Mayo de 2012, de https://www.icbf.gov. co/icbf/directorio/portel/libreria/pdf/LINEAMIENTOSLINEAMIENTOSHOGARESINFANTILESLACTANTESYPREESCOLARES.pdf.

Íñiguez Rueda, L. \& Antaki, C. (1994). Análisis del discurso en psicología social. Boletín de Psicología, 44, 57-75.

Macías, A., Amar, J., \& Jiménez, M. (2005). Dinámica de las familias de menores con problemas psicosociales: el caso del menor infractor y la menor explotada sexualmente. Revista Latinoamericana de Ciencias Sociales, Niñez y Juventud, 3(2), 141-174.

Ministerio de Protección Social MPS (2006). Lineamientos de Intervención Psicosocial con la población en condición y situación de desplazamiento por la violencia en Colombia 2006-2010. Recuperado el 10 de Octubre de 2012 de http://www.disaster-info.net/desplazados/informes/pah2/sesion040/MPS\%20 Versi\%F2n\%20011106.pdf

Molina, N, (2012). Panel Qué es lo psicosocial ¿otra vez la misma pregunta? o ¿la misma respuesta? Ponencia en el 1er Encuentro Nacional y Cátedra Internacional sobre Intervenciones Psicosociales-FUNLAM-Medellín.

Montenegro, M (2001). Conocimientos, Agentes y Articulaciones: Una mirada situada a la intervención social. Tesis Doctoral. En: http://hdl. handle.net $/ 10803 / 5410$.

Montero, M. (2003). Teoría y práctica de la psicología comunitaria: La tensión entre comunidad y sociedad. Buenos Aires: Paidós.

Montero, M. (2004). Relaciones Entre Psicología Social Comunitaria, Psicología Crítica y Psicología de la Liberación: Una Respuesta Latinoamericana. Psykhe, 13(2), 17-28.

Montero, M (2004). Relaciones entre Psicología Social Comunitaria, Psicología Crítica y Psicología de la Liberación: Una Respuesta Latinoamericana. 
Moscovici, S (1986). Psicología Social I. Influencia y Cambios de Actitudes; Individuos y grupos. Barcelona, España: Paidós.

Muñoz, J. (2004). Análisis cualitativo de datos textuales con ATLAS.ti 5. Universitat Autònoma de Barcelona. Juan Muñoz Justicia@. Disponible en: http://www.geocities.com/cibertlan/ docs/materiales/ManualAtlas5.pdf [consulta 2013, 22 de abril]

Musitú, G. (dir.) (1993). Intervención psicosocial: programas y experiencias. Madrid. Editorial Popular.

Rincón, H. G., Castillo, A., Reyes, C., Toro, C. E., Rivas, J. C., ... Ocampo, A. (2006). Atención psicosocial integral en enfermedad catastró- fica (Atinar). Atención psicosocial integral en enfermedad. Revista Colombiana de Psiquiatría, S35, 44-71.

San Juan, C. (Coord.) (1996). Intervención Psicosocial. Elementos de programación y evaluación socialmente eficaces. Bogotá: Editorial Anthropos.

Vasco, C. (2012). Experiencias y Sentidos del Trabajo Psicosocial. Tesis de Maestría. Universidad de los Andes, Facultad de Ciencias Sociales, Departamento de Psicología.

Vázquez, F. (1994). Análisis de contenido categorial: "el análisis temático" Unitat de Psicología Social. Universitat Autónoma de Barcelona. 